\title{
Stress and coping among children of alcoholic parents through the young adult transition
}

\author{
Andrea M. Hussong ${ }^{a}$ and Laurie Chassin ${ }^{b}$ \\ aUniversity of North Carolina at Chapel Hill \\ ${ }^{\mathrm{b}}$ Arizona State University
}

\section{Abstract}

The transition to young adulthood is both a time when risky health behaviors such as substance misuse peak and a time of opportunity for growth and development through the acquisition of adult roles. In this transition, coping styles include responses to the stressors and opportunities associated with the emergence of adulthood. The extent to which such coping styles are skillfully employed in part determines adjustment into adulthood. The current study used a high-risk, longitudinal design to examine the development of coping styles over adolescence, continuity in these coping styles from adolescence to adulthood, the impact of coping on adult stress and substance misuse, the ability of coping to buffer effects of stress on substance use, and differences in coping between at-risk youth (i.e., children of alcoholics [COAs]) and their peers. A sample of 340 adolescents completed four assessments over ages 11-23. We used latent trajectory models to examine interindividual and intraindividual change in coping over time. Evidence for both change and continuity in the development of coping from adolescence to adulthood was found, although adolescent coping had limited impact on stress and substance use in adulthood. Support was also found for complex stress-buffering and stress-exacerbating effects of coping on the relations between major life events and adult drug use and between stress associated with the new roles of adulthood and heavy alcohol use. Implications of these findings for development and adjustment in the transition to adulthood are discussed.

The role changes and demands associated with the transition to adulthood present developmental challenges offering opportunities for change and growth. From a developmental-contextual perspective, how individuals respond to these challenges, in turn, impacts their adjustment and symptomatology into adulthood (Lerner, Lerner, von Eye, Ostrum, Nitz, Talwar-Soni, \& Tubman, 1996). These responses include personal and social assets that facilitate coping, a critical determinant of successful functioning during this period of life (Eccles, Templeton, Barber, \& Stone, 2003). As such, the coping skills that adolescents bring into young adulthood and how these skills are used to address concomitant developmental stressors are key for understanding successful negotiation of the transition to adulthood.

Peak levels of substance use and misuse coincide with this developmental period (Bachman, Wadsworth, O'Malley, Johnston, \& Schulenberg, 1997) and serve as one domain for identifying the potential for failures in negotiating this transition. Although in many instances the acquisition of adult roles is associated with lower substance involvement (Bachman et al., 1997), high levels of alcohol and drug use also predict premature entry into

Copyright (C) 2004 Cambridge University Press

Address correspondence and reprint requests to: Andrea Hussong, Department of Psychology, CB\#3270, Davie Hall, University of North Carolina at Chapel Hill, Chapel Hill, NC 27599-3270; ahussong@email.unc.edu. 
adult roles (Newcomb \& Bentler, 1988; Newcomb, 1996) as well as more difficulty in and early disruption of these roles (i.e., lower work status, job problems, lower degree attainment, unemployment, divorce; Bachman et al., 1997; Gotham, Sher, \& Wood, 2003; Newcomb \& Bentler, 1985, 1986; Stein, Smith, Guy, \& Bentler, 1993).

A group at particular risk for greater substance use and problematic sequelae during young adulthood is children of alcoholic parents. During adolescence, greater stress partly accounts for elevated rates of alcohol and drug use for this group, suggesting the potential for an etiological role of stress in early phases of substance use (Chassin, Curran, Hussong, \& Colder, 1996). The extent to which stress in the transition to young adulthood also accounts for drinking and drug taking during this peak period of risk is expected to vary across individuals, with coping skills serving to protect vulnerable individuals from misusing substances. To address these issues, the current study examined the extent to which coping skills differ for COA versus nonalcoholic parents, show greater continuity or change from adolescence to young adulthood, predict experiences of young adulthood including stress and substance use, and serve to protect young adults exposed to greater stressors from substance misuse.

\section{The Development of Coping}

As often cited, Lazarus and Folkman (1984, p. 141) define coping as "constantly changing cognitive and behavioral efforts to manage specific external and/or internal demands that are appraised as taxing or exceeding the resource of the person." Many different operationalizations of coping have appeared in the literature since this definition was first offered, and recent reviews have continued ongoing debates as to the limits of what qualifies as coping (i.e., vs. symptomatology or in terms of voluntary vs. involuntary responses to stress) and the structure that is most useful in distinguishing among coping efforts (Ayers, Sandler, \& Twohey, 1998; Compas, Connor-Smith, Saltzman, Thomsen, \& Wadsworth, 2001). The myriad conceptualizations of coping currently make explication of a single agreed upon operationalization of coping impossible; however, several popular distinctions have emerged. The first is between coping styles, which delineate typical responses to stress over prolonged periods of time, and coping strategies, which delineate moment to moment efforts to address a particular stressor in a particular context (Sandler, Wolchik, MacKinnon, Ayers, \& Roosa, 1997). Coping strategies permit greater exploration of variability in coping responses as a function of stressors and context as well as a clearer elaboration of the coping process itself. However, the cumulative impact of strategies on longer term development are perhaps better conveyed through the study of coping styles, reflecting the habits of coping. Given our focus on the development of coping and its impact on adjustment from adolescence to adulthood, the current study examined coping styles.

In an early effort to define coping styles in adolescence, Wills $(1985,1986)$ developed a behavior-based measure of cognitive (i.e., "representing minimization of distress, selective focus on positive aspects, and positive comparisons," p. 509) and behavioral coping (i.e., "active approaches to information gathering, decision making and problem solving," p. 509). Factor analyses of the original 54-item measure revealed 11 factors, including factors labeled behavioral and cognitive coping that most closely resemble the original two content domains. Wills reported no information on the reliability of the scales, but did show factor structure stability in the measure across four waves of data, beginning with seventh and eighth grade adolescents, as well as concurrent validity in which behavioral and cognitive coping were directly and inversely related to substance use and served as moderators of the relation between stress and substance use. 
Subsequent studies have also made the distinction between cognitive and behavioral coping in adolescence (Compas et al., 2001), although Ayers et al. (1998) question whether the cognitive styles characterized by positive restructuring (i.e., "Try to notice only the good things in life") versus cognitive avoidance (i.e., "Try to put it out of my mind") may show different relations to outcomes. Drawing on a conceptually similar comparison, Compas et al. (2001) offer the general conclusion that coping styles characterized as engaged, problem solving, and behavioral are associated with more positive outcomes than are those characterized as disengaged or avoidant.

The current study adapts the scales developed by Wills $(1985,1986)$ to study coping in the transition to adulthood, adopting the terms planful or behavioral coping and avoidant or cognitive coping to more clearly identify the content of these scales. This distinction among coping styles has the advantages of mapping onto previous literature with adolescents and adults regarding risk for substance use, is drawn from a long tradition of distinguishing between coping styles based on the domain of coping, and is related to an established measure developed for adolescents, which is useful in examining change and continuity between adolescence and young adulthood.

The psychopathology literature distinguishes between homotypic continuity (i.e., stability in the form of a given behavior over time) and heterotypic continuity (i.e., stability in the underlying construct despite change in its observed manifestations over time; Cicchetti, Rogosch, \& Toth, 1997; Sameroff, 2000). In the current study, we focus on the former (given poor consensus in the coping field on what might form the underlying construct of coping in the absence of consistency in manifestations), and we distinguish between two forms of such continuity; namely, interindividual and intraindividual continuity. Interindividual continuity focuses on stability over time across individuals, where those higher in coping at one time point remain higher in coping relative to their peers at later time points. Intraindividual continuity focuses on stability over time within individuals, where high scores at one time point are maintained within person as high scores for that same individual at a later time point. Focusing on interindividual stability, previous studies have examined mean differences and rank-order stability (i.e., via correlational methods) in similar forms of coping over time. Reviewing this literature, Compas, Banez, Malcarne, and Worsham (1991) noted consistent support for increases in emotion-focused coping (which is often related to avoidant or cognitive forms of coping) across childhood and adolescence. Findings for problem-focused coping were more inconsistent, with several studies finding no change over time, and some even showing decreases with age. Many of the studies contributing to this literature, however, provide cross-sectional age comparisons. Moreover, results from longitudinal studies are based on interindividual comparisons of change that do not permit conclusions about intraindividual patterns of dynamic change. The latter are of greater interest to us in that we propose individual differences in the development of coping, such that not all adolescents experience changes in coping over time in a uniform manner.

The potential for such intraindividual differences in development is evident from the very mechanisms that may support developmental changes in coping more generally. As reported by Ayers et al. (1998) and Losoya, Eisenberg, and Fabes (1998), reasons for change in coping throughout adolescence include the acquisition of more complex cognitive processing strategies, the development of meta-cognition, changes in the desired and available sources of social support, shifts in desired sources and forms of coping assistance, gains in perspective taking and experience that lead to changes in stress appraisal, and changes in the meaning of behaviors that may change the function of a given coping response over time. Social and cognitive developmental gains underlie most of these mechanisms purported to propel changes in coping over time. However, evidence of individual variation in rates of cognitive and social development over time would, in turn, 
suggest that individual adolescents and budding adults vary from one another in the development of their coping styles as well.

\section{Coping and Substance Use}

Coping styles are purported to have both direct and moderated effects on substance use and misuse (Maisto, Carey, \& Bradizza, 1999; Wills, 1986). Stress-buffering and stressexacerbating theories of coping capture the effects of coping as a moderator of the relation between stress and substance use. Previous studies of young adults have found mixed support for the stress-buffering hypothesis, with no effects found in many studies (e.g., Cooper, Russell, \& George, 1988; Laurent, Catanzaro, \& Callan, 1997; Windle \& Windle, 1996) and small effects of problem-focused or planning coping to dampen the relation between stress and alcohol use found in others (e.g., Wills, Sandy, Yaeger, Cleary, \& Shinar, 2001). Stress-exacerbating effects are more common, with greater avoidance and emotion-focused coping strengthening the association between stress and drinking (e.g., Cooper et al., 1988; Laurent et al., 1997; Wills et al., 2001).

Findings concerning the direct effect of coping on substance use in young adulthood are more mixed. The anticipatory use of coping skills may reduce risk for substance misuse directly by reducing the subjective experience of stress, by limiting exposure to stress or appraisal of encountered stressors, or by increasing preparedness for, confidence in, or time for choosing alternative strategies for responding to stress (Wills et al., 2001). As such, coping may directly impact both stress and substance misuse such that adolescent coping skills predict the experience of stress and engagement in alcohol and drug use in young adulthood.

Fewer studies have investigated the impact of coping on stress directly, although some support for this association has been reported in the literature (Wills, 1989; cf. Sandler, Tein, \& West, 1994). Receiving greater research attention, direct effects of coping on substance use show that active and problem-focused styles are often inversely related (Wills, 1986; Wills et al., 2001; Windle \& Windle, 1996) or unrelated to alcohol involvement (Cooper, Russell, Skinner, Frone, \& Mudar, 1992; Fromme \& Rivet, 1994), whereas avoidant, emotionfocused styles generally predict greater alcohol use (Cooper et al., 1992; Fromme \& Rivet, 1994; Wills et al., 2001; Windle \& Windle, 1996). Inconsistent relations between cognitive coping and substance use have also been found (Wills, 1986; Wills et al., 2001). Although direct comparisons of these models for the prediction of alcohol versus drug use are rare, previous research suggests that coping reasons for use are more strongly associated with problematic outcomes (i.e., the use of drugs and binge drinking) compared with more normative alcohol use (Cooper, Frone, Russell, \& Mudar, 1995).

\section{Stressors in Young Adulthood}

Not all stressors are equally salient predictors of substance misuse. Daily life hassles, as opposed to major life events, are more strongly related to poor health outcomes in adults (Compas, Howell, Phares, Williams, \& Giunta, 1989; DeLongis, Coyne, Dakof, Folkman, \& Lazarus, 1982; Wagner, Compas, \& Howell, 1988), although this is not necessarily true for adolescents (Baer, Garmezy, Mclaughlin, Pokorny, \& Wernick, 1987; Wills, 1986). For adolescents, studies have found stronger relations between substance use and major life events than between substance use and daily hassles. Finally, negative, major life stressors have been more strongly linked to substance use than have positive events (Wills, 1986).

One distinction that has, however, not been examined is that between more general life stressors and those linked to developmental transition. The stress of the developmental transition to young adulthood may, in part, be defined with respect to new roles. Arnett's 
(2000) research defines the goals or tasks of the transition to young adulthood in terms of identity development, involving the pursuit of love, work, and world views. Young adults themselves primarily define the tasks of this transition in terms of gaining independence and accepting responsibility for one's actions, although sociologists tend to focus on role acquisition related to employment or advanced education, marriage, and parenthood as markers of this transition (Arnett, 2001). Indeed, role acquisition may, in part, hasten the transition to adulthood through associated opportunities for independence and responsibility.

Although studies of adult roles and substance use primarily show a decrease in use associated with acquiring these new roles (e.g., Bachman et al., 1997), Todd, Chassin, Presson, and Sherman (1996) report that among young adults who are already in these roles, role-related stress predicts greater cigarette use. We posit that such role or transition-related stress may have a unique relation to substance use from that of major life events. Although transition-related stressors are similar to negative, major life stressors in that each vary in terms of their impact on daily living, how much they tax coping resources, and their perceived controllability, transition-related stress differs from major life events on five important points.

First, compared to negative, major life events, transition-related stressors are more likely to be socially embedded. Young adults seek out friends to talk about romance, work, and identity, gaining support from a peer who is facing the same developmental challenges (Carbery \& Burhmester, 1998). However, peer support in young adulthood may also provide opportunity for engaging in other socially mediated experiences that convey a sense of pseudomaturity, like substance use (Newcomb, 1996). Indeed, previous studies show that in adolescence and young adulthood, social support from friends is actually a positive predictor of substance involvement (e.g.,Wills, 1986).

Second, in contrast to negative, major life events, transition-related stressors include both positive and negative experiences associated with the tension between gaining autonomy and gaining responsibility (Bachman et al., 1997). The complex demands of such tensions may call for more complex coping strategies that include both aspects of cognitive and behavioral coping.

Third, unlike major life events that are largely unexpected, transition-related stressors represent entry into roles that are much anticipated by emerging adults. Indeed, through play, children begin practicing and shaping their expectations for these roles from an early age. Such anticipations may both ease the transition into these roles, providing ample time for cognitive preparation, but also interfere with a realistic expectation and subsequent appraisal of the new role.

Fourth, transition-related stressors are, in part, defined by their likely contribution to the central task of this period, identity development (Arnett, 2000). For this reason, avoidant forms of coping, for example, may be particularly deleterious by signifying the potential for delay in developmental gains. The impact of new roles on identity may imbue otherwise less meaningful daily hassles with greater personal meaning, increasing their salience, their negativity, and their impact on the individual.

Fifth, unlike major life events, transition-related stressors are linked to role changes that have a pervasive impact on young adults. Social structures, interpersonal social influences, and individual characteristics as identified in many prominent theories of drug use may all be impacted by these role changes (Bachman et al., 1997). As a result, stress in these new roles may signify change in the proximal risk structure for substance involvement. 
These five points distinguish the experience of major life events and transition-related stressors. In addition, they highlight the potential for these two forms of stress to differentially impact substance involvement. Although we anticipate that the relation between both forms of stress and substance use will be moderated by coping strategies, differences in the stressors themselves may make different forms of coping relevant as stress buffers or exacerbators of risk for substance use. We tested this hypothesis in the current study.

\section{The Current Study}

A developmental focus on stress and coping models in young adulthood compels us to address two important weaknesses in the literature concerning adolescent and young adult coping. First, very few studies employ longitudinal designs that permit conclusions about the development of coping behavior over time. Although several studies suggest that different forms of coping may emerge over adolescence, available studies have largely used age-graded cross-sectional samples to infer ontogeny. In the current study, we use a longitudinal design to examine developmental patterns of coping over adolescence and to test whether these patterns are predictive of coping styles in young adulthood. Moreover, we extend these models to consider the impact of adolescent patterns of coping on young adult outcomes and whether coping in young adulthood buffers the potentially deleterious effects of general life and transition-specific stress during this period.

Second, few studies include high-risk groups to increase variation in the types of coping and adjustment outcomes observed. In the current study, we consider the development of coping, stress, and substance involvement in a sample of COAs and matched controls. Compared to their peers, COAs consistently show greater risk for substance use and misuse and higher rates of life stress both in adolescence and young adulthood (Chassin, Rogosch, \& Barrera, 1991; Sher, 1991; Sher, Walitzer, Wood, \& Brent, 1991). Although maladaptive or immature coping strategies are speculated to contribute to such negative outcomes, little research has examined the development of coping in this risk group. Related literature suggests that COAs are more likely to use anger as a defense mechanism (Jarmas \& Kazak, 1992) and that individual escape-avoidance coping predicts greater symptomatology among COAs (Easley \& Epstein, 1991) as well as that COAs do not differ from their peers in coping styles (Johnson \& Pandina, 1993; Kelly \& Myers, 1997) and that COAs' risk for negative outcomes are not buffered by coping or social support (Menees, 1997). Thus, this brief literature has produced inconsistent results.

In sum, the current study examined the hypothesis that coping styles impact the relation between stress and substance use in young adulthood, with the origins of these coping skills lying in adolescence. Using a prospective, high-risk design, we addressed four specific questions about the development and impact of coping in young adulthood.

1. How do trajectories of coping over adolescence relate to coping styles in young adulthood?

2. Are those young adults with greater coping skills prior to young adulthood at lower risk for substance use and stress (either generally or with respect to transition specific stressors) in young adulthood?

3. Do young adult coping styles buffer the relations between young adult stress (both general life stress and transition-specific stress) and substance use?

4. How do these coping styles and their impact on substance use differ for COAs and their peers? 


\section{Method}

\section{Sample and procedure}

A community sample of 454 families completed three annual interviews when the target child was an adolescent (at ages 11-16, 12-17, and 13-18 across waves) and a follow-up interview when the target was a young adult (5 years after Wave 3 at ages 18-23). Alcoholic parents were identified through court records, HMO wellness questionnaires, and telephone surveys. Inclusion criteria for COA families were living with a biological child aged 11 15, non-Hispanic Caucasian, or Hispanic ethnicity, English speaking, and a biological and custodial parent who met DSM-III lifetime criteria for alcohol abuse or dependence. Control families were matched to these COA families on the basis of ethnicity, family structure, socioeconomic status (SES), and the adolescent's age and gender. Attrition biases were minimal, with $97 \%$ subject retention over the first three waves of assessment and $90 \%$ retention of the initial Wave 1 participants at the young adult follow-up (for details, see Chassin, Barrera, Bech, \& Kossak-Fuller, 1992; Chassin, Pitts, DeLucia, \& Todd, 1999). Although participants completed computer-based interviews at each wave, to shorten the assessment battery in young adulthood some measures (such as the life events and coping measures used here) were completed as part of a survey that participants mailed back to researchers outside of the interview session. A total of $88 \%$ of participants completing the Wave 4 battery returned these surveys.

Only participants with complete data on relevant outcome measures in young adulthood were retained for analyses, leaving a sample of 340 participants (51\% male, 51\% COAs, $74 \%$ non-Hispanic Caucasian, 26\% Hispanic, 11-15 years old at Wave 1, mean age $=12.7$ ). Chi-square and $t$ test analyses tested attrition effects on all demographic measures as well as coping, stress and drug use at Waves 2-4. Of these 16 comparisons, those retained in the current sample differed from attriters in five instances; sample attriters were more likely to be COAs, $\chi^{2}(1, N=454)=4.94, p=.03$, male, $\chi^{2}(1, N=454)=6.47, p=.01$, and to report more life stressors, unbalanced: $t(1,163)=-2.27, p<.05$, and greater substance use at Waves 3: $t(1,144)=-2.68, p<.01$, and $4: t(1,86)=-1.95, p=.05$. These attrition effects are further examined in post hoc analyses for each model.1

\section{Measures}

Psychometric properties, univariate statistics, and correlations among variables of interest are reported in Table 1. With the exception of parent diagnoses, all variables were assessed by the target adolescent's self-report.

Parent diagnoses-Families were classified as alcoholic if either parent met DSM-III lifetime criteria for diagnoses of alcohol abuse or dependence. When possible, parents were directly interviewed using a computerized version of the Diagnostic Interview Schedule III (Robins, Helzer, Croughan, \& Ratcliff, 1981) to assess diagnostic status. In cases where a biological parent was not directly interviewed ( $21 \%$ of fathers and $4 \%$ of mothers in the current subsample), the reporting parent was used as the informant using the family history research diagnostic criteria (Andreasen, Endicott, Spitzer, \& Winokur, 1977) to assess

\footnotetext{
${ }^{1}$ All primary analyses were estimated using missing data algorithms available through MPLUS to determine the effects of attrition. These reanalyses provided support to all effects reported for analyses using listwise deletion. However, additional effects were also found. For example, analyses testing hypothesis 1 (predicting young adult coping) showed significant variability in the slope of planning coping over time that, in turn, predicted greater active coping in young adulthood. In analyses of the second hypothesis, adolescent life stress predicted young adult transition-related stress in both models of heavy alcohol use and drug use. Overall, these analyses suggest little effect of attrition on substantive conclusions. Note that because we are unable to determine whether role stress is a relevant variable for individuals with missing data at Wave 4 (i.e., because we do not know their role occupancy), we chose to be conservative in using these missing data routines and to present the listwise deletion analyses as our primary findings in text.
} 
alcoholism. Previous studies have found a high degree of test-retest reliability using this method to diagnose alcoholism (98.8\% agreement, $\kappa=.95$; Zimmerman, Coryell, Pfohl, \& Stangl, 1988) and excellent specificity (92\%) and sensitivity (90\%) for wives reporting on their husbands' substance abuse disorders (Kosten, Anton, \& Rounsaville, 1992). In the current sample, $53 \%$ of families had at least one alcoholic parent.

Adolescent coping-Thirteen items from a coping measure developed by Wills (1986) were designed to assess behavioral (e.g., "When I have a problem, I change a behavior that contributes to the problem") and cognitive (e.g., "When I have a problem, I try to go on as if nothing had happened") coping. Due to low reliability estimates for these subscales as originally scored by Wills, we conducted exploratory factor analyses of these items using promax rotation at the first three measurement waves. Analysis of the scree plot and eigenvalues (following Loehlin, 1992) indicated that two factors best represent the items; the first reflected planful or behavioral approaches to coping (three items), and the second reflected cognitive approaches to coping (including items classified as both cognitive and more avoidant forms of coping on previous measures; nine items). A mean of each subscale formed the coping variables at each of the three waves of measurement during adolescence.

Young adult coping-To expand the assessment of coping in young adulthood, a new measure of coping was introduced in the young adult battery. At Wave 4, 24 items taken from Carver, Scheier, and Weintraub (1989) and Zautra, Sheets, and Sandler (1996) assessed the three broad dimensions of active coping (including items assessing active, planning, and restraint coping), cognitive coping (including items assessing positive reinterpretation, humor, and acceptance), and avoidant coping (including items assessing denial and mental disengagement). Participants rated how often they used each coping strategy when faced with a stressful event on a 4-point scale ranging from I usually don't do this at all to I usually do this a lot. Exploratory factor analyses of these items in the current study confirmed this three factor structure, and a mean of items on each subscale formed the three young adult coping variables.

Adolescent and young adult life stress events-Seventeen age-appropriate items administered in both adolescence and young adulthood formed the life stress measures for the current study. These items were drawn from the Children of Alcoholics Life Events Schedule (Roosa, Sandler, Gehring, Beals, \& Cappo, 1988), General Life Events Schedule for Children (Sandler, Ramirez, \& Reynolds, 1986), and other life events schedules for children and adolescents. Items were coded as having occurred (1) or not (0). An average of reported events that occurred within the past 3 months served as the life events indicator.

Young adult transition-related stress-Following Todd et al. (1996), we focused on stress rather than occupancy within four roles associated with the transition into young adulthood; namely, being a postsecondary student, employee, marital partner, and parent. Only four participants of those with complete data on Wave 4 measures did not occupy one of these four roles, and were thus omitted from analyses. Within the 5 years preceding the Wave 4 interview, 17\% of the remaining 340 participants were parents (who either did or did not live with their children), $68 \%$ had been married or in serious romantic relationships, $80 \%$ had held full- or part-time paying, non-temporary jobs, and $75 \%$ had been full- or parttime students. Only participants in a particular role within the past 5 years reported on items regarding stress in that role.

Five items each were modified from the decision authority subscale (Schwartz, Pieper, \& Karasek, 1988) to assess work and school role demands (e.g., "In the past year, how much did your job require you to work fast"). Participants rated the extent to which each demand was stressful for them on a 5-point scale ranging from not at all to a great deal. Cronbach's 
alpha estimates were .81 and .80 for the student and work stress scales, respectively. Four items assessing parenting stress and three items assessing marital stress were adapted from Todd et al. (1996; e.g., "In the past year, I had difficulties arranging for childcare"). Participants rated the frequency with which these role demands occurred using a 5-point scale ranging from never to very often. Cronbach's alpha estimates were .80 and .83 for the parenting and marital stress scales, respectively.

Because the goal of the current study was to examine whether stress associated with young adult roles in general, and not with respect to any one role in particular or in contrast to another, was associated with substance involvement and because we wanted to retain participants in a single analysis when possible, we created one index of transition-related stress from these four measures of role stress. Averages of available reports of role stress across the four roles for each participant were formed for this purpose. Note that low correlations between transition-related stress and repeated measures of general life events (see Table 1) suggest that these constructs are independent.

Adolescent and young adult substance use-Participants self-reported their frequency of substance use in the past year using an 8-point response scale (from none to daily) on 12 items, including drinking beer, wine, and hard liquor; drinking five or more drinks in a row; getting drunk on alcohol; and using eight illicit drugs (Chassin et al., 1991). To control for overall substance involvement in adolescence, we created an adolescent substance use measure from the maximum frequency of alcohol or any of the eight illicit substances at Wave 3. For our young adult outcome variable, we separated heavy alcohol use (i.e., the summed frequencies of getting drunk or drinking five or more in a row) and drug use (i.e., the summed frequencies of using any of the eight illicit drugs) because the differential normativeness of alcohol and drug use in emerging adulthood might yield differing predictors.

\section{Results}

We used latent trajectory modeling and hierarchical regression analyses to test four questions in the current study. Psychometric properties for each variable used in these analyses, as well as correlations among these variables, are reported in Table 1. All latent trajectory models (LTMs) were estimated using MPLUS. Model fit was assessed using the chi-square test statistic, the Tucker-Lewis index (TLI; Tucker \& Lewis, 1973), the comparative fit index (CFI; Bentler, 1990), and the root mean standard error of approximation (RMSEA; Browne \& Cudeck, 1993; Steiger \& Lind, 1980). CFIs greater than .90 and an RMSEA less than .05 were taken to indicate acceptable fit.

\section{Relations among parent alcoholism, adolescent coping, and young adult coping}

As reported in Table 1, bivariate correlations suggested continuity over time in cognitive coping ( $r=.35-.47$ across Waves 1-3 measures) and in planning coping $(r=.39-.52)$, with effect size estimates in the small to medium range $\left(r^{2}=.12-.27\right)$. Means of the adolescent coping measures also show decrements from Wave 1 to Waves 2 and 3 in both forms of coping. These adolescent measures showed a fairly consistent pattern of correlations with young adult coping, with positive associations between adolescent planning and young adult active coping $(r=.18-.27)$ and between adolescent cognitive and young adult avoidant coping $(r=.20-.21)$ as well as negative associations between adolescent planning and young adult avoidant coping $(r=-.18$ to -.22$)$. The associations from adolescent to adult coping measures, however, are consistent with small effect sizes $\left(r^{2}=.04-.07\right)$. These bivariate correlations support previous findings of small to moderate interindividual continuity in coping over time, and also show possible decrements in coping over time. 
To more rigorously test intraindividual patterns of change in coping, we next used LTMs. Our initial model included adolescent age, gender, and parent alcoholism as exogenous predictors of latent trajectories underlying change in planning and cognitive coping across adolescence. Given the three time points available for these analyses, linear trajectories were estimated for each construct. Results from this model indicated an excellent fit to the data: $\chi^{2}(10, N=340)=11.91, p=.29, \mathrm{CFI}=1.0, \mathrm{TLI}=0.99, \mathrm{RMSEA}=.02$, confidence interval $(\mathrm{CI})=.00-.07$. Intercepts for both cognitive $(M=3.56, z=13.76)$ and planning $(M=4.34, z$ $=11.92$ ) coping were significantly different from zero, and varied significantly across individuals ( $\psi=.17, z=5.61$ and $\psi=.31, z=5.25$, respectively). The average slope for both of these trajectories was decreasing over time $(M=-0.42, z=-2.80$ for cognitive coping and $M=-0.65, z=-3.05$ for planning coping) and estimates of individual variability within the sample were significant for cognitive coping $(\psi=.03, z=2.17)$ and marginally significant for planning coping $(\psi=.06, z=1.88)$. These results suggest that estimates of average cognitive coping began at 3.56 atWave 1 and dropped to 2.72 at Wave 3 and that average planning coping began at 4.34 at Wave 1 and dropped to 3.04 at Wave 3.

To test whether these trajectories of coping over adolescence predict coping behavior during young adulthood, we expanded our LTM analysis to include the Time 4 outcomes of active, avoidant, and cognitive coping. Because adolescent coping was also associated with both life stress and substance use in adolescence (at Wave 3; see Table 1), we controlled for these potential confounds for a more stringent test of continuity in coping over time. 2 As such, Time 3 assessments of life stress and substance use were included in the model as endogenous mediators along with our latent trajectory factors for both adolescent coping scales. All contemporaneous variables were freely correlated and paths for all variables assessed at earlier time points to those at later time points were estimated, with the exception that no direct pathways to the adolescent coping variables were estimated as all effects of adolescent coping were modeled through the latent trajectory factors. The final estimated model is depicted in Figure 1.

The resulting model provided an excellent fit to the data, $\chi^{2}(16, N=329)=17.42, p=.36$, $\mathrm{CFI}=1.0, \mathrm{TLI}=0.99, \mathrm{RMSEA}=.02, \mathrm{CI}=.00-.05$. Results for pathways of key interest are reported in Table 2. Greater levels of planning coping in early adolescence predicted greater active coping in young adulthood $(\beta=.36, z=4.50, p<.001)$. Moreover, greater levels of adolescent cognitive coping $(\beta=.26, z=3.35, p<.001)$ and lower levels of adolescent planning coping $(\beta=-.27, z=-3.59, p<.001)$ predicted greater young adult avoidant coping. Finally, higher levels of adolescent planning coping were marginally associated with higher cognitive coping in young adulthood $(v=.16, z=1.68, p=.10)$. No associations were found for Time 3 stress and substance use with Time 4 coping outcomes. These results suggest continuity in behavioral (i.e., planning and active) coping over time and in avoidant coping over time. The final model accounted for 15,18 , and $9 \%$ of the total variance in Wave 4 active, avoidant, and cognitive coping, respectively.

Associations between coping and parent alcoholism were also tested in this model. COAs were marginally less likely to report active coping in young adulthood as compared to their peers $(\beta=-.13, z=-1.79, p<.10)$, but no differences in young adult cognitive or avoidant coping were found. Though not reported in Table 2, marginal effects suggested that, in

\footnotetext{
${ }^{2}$ We estimated all primary analyses with and without the presence of the Wave 3 control variables of adolescent substance use and major life stress. Results did not differ in analyses of hypothesis 1 (predicting young adult coping outcomes). However, for analyses of hypothesis 2 , lower levels of planning coping in early adolescence predicted greater major life stress in young adulthood $(\beta=-.02, z=$ $-2.17, p<.05$ in the alcohol use model and $\beta=-.15, z=-2.19, p<.05$ in the drug use model), a marginal effect of greater levels of cognitive coping in adolescence predicted greater transition-related stress in young adulthood $(\beta=.13, z=1.76, p<.10$ in the alcohol use model and $\beta=.13, z=1.77, p<.10$ in the drug use model), and parent alcoholism was marginally associated with drug use $(\beta=$. $11, z=1.76, p<.10)$ and significantly associated with heavy alcohol use $(\beta=.13, z=2.08, p<.05)$.
} 
adolescence, COAs reported higher levels of cognitive $(\beta=.12, z=1.70, p<.10)$ and lower levels of planful $(\beta=-.13, z=-1.83, p<.10)$ coping than did their peers.

\section{Relations between adolescent predictors and young adult stress and substance use}

This set of analyses tested whether adolescent coping, life stress, and substance use predicted stressful life events, transition-related stress, and substance use in young adulthood. The LTM approach used to test the first hypothesis (see Figure 1) was modified for the current analysis and the resulting model is depicted in Figure 2. Age, gender, and parent alcoholism served as exogenous predictors of the two coping trajectories in adolescence as well as of adolescent life events and substance use. The control variables of adolescent life stress and substance use were included in this model to more stringently test for effects of adolescent coping on young adult stress and substance use above and beyond continuity in these outcomes over time (see footnote 2). Separate models were estimated for the outcomes of heavy alcohol use and drug use, given their different prevalence and thus ability to serve as indicators of deviance in young adulthood.

The resulting models fit the data well: $\chi^{2}(16, N=340)=24.58, p=.08, \mathrm{CFI}=0.99$, TLI $=$ 0.93 , RMSEA $=.04, \mathrm{CI}=.00-.07$ for heavy alcohol use; $\chi^{2}(16, N=340)=17.61, p=0.35$, $\mathrm{CFI}=1.0, \mathrm{TLI}=0.98, \mathrm{RMSEA}=.02, \mathrm{CI}=.00-.05$ for drug use. Results for pathways of particular interest are reported in Table 3. For the model predicting heavy alcohol use, continuity in major life stress was found with Wave 3 life stressors predicting major life stress in Wave $4(\beta=.20, z=3.39, p<.001)$. No other predictors of young adult major life stress or of transition-related stress were found. Males $(\beta=.29, z=2.66, p<.001)$, those reporting greater substance use in late adolescence $(\beta=.31, z=2.93, p<.001)$, and those with lower levels of planning coping in early adolescence $(\beta=-.20, z=-2.18, p<.05)$ all reported greater heavy alcohol use than their peers. In the model predicting drug use, the effects of Wave 3 stressors on Wave 4 major life events was again evident $(\beta=.20, z=3.32$, $p<.001)$ and lower planning coping in early adolescence also predicted more frequent drug use $(\beta=-.16, z=-2.29, p<.05)$.

Effects of parent alcoholism on these young adult outcomes were again estimated in these models. However, after controlling for substance use at Wave 3, no further effects of parent alcoholism were evident although COAs did report greater levels of both alcohol $(\beta=.21, z$ $=4.00, p<.001)$ and drug $(\beta=.19, z=3.60, p<.001)$ use at Wave 3 .

\section{Stress, coping, and problem behaviors in young adulthood}

In the final analyses, we examined the buffering effects of young adult coping on the relation between stress and substance use. Because this hypothesis did not involve latent factors or growth modeling, we tested this hypothesis using three hierarchical regression analyses. Separate models were again estimated for heavy alcohol and drug use. These models predicting substance use included parent alcoholism and control variables (age, gender, and Time 3 substance use) in Step 1, the three forms of young adult coping in Step 2, the two forms of stress in Step 3, and the interactions between each form of coping and stress in Step 4 (see Table 4).3 Both models accounted for significant variance in the outcomes, $F(15,324)=5.85, p<.001$, adjusted $R^{2}=.18$ for heavy alcohol use and $F(15$, $285)=3.01, p<.001$, adjusted $R^{2}=.08$ for drug use.

\footnotetext{
${ }^{3}$ Because of significant age heterogeneity within wave, we tested whether the Stress $\times$ Coping interactions varied as a function of age in predicting substance use outcomes. Regression analyses were reestimated including Age $\times$ Stress $\times$ Coping interactions and all relevant two-way interactions, resulting in a total of six three-way interactions per model. None of the three-or two-way interactions involving age were significant.
} 
In the alcohol use model, males, $\beta=.21, t(1,324)=4.21, p<.001$; COAs, $\beta=.13, t(1$, $324)=2.46, p<.01$; and those who reported greater substance use at Wave $3, \beta=.28, t(1$, $324)=5.23, p<.0010$ or greater major life stress at Wave $4, \beta=.13, t(1,324)=2.47, p<$. 001 , all reported more frequent heavy alcohol use than their peers. No main effects of coping or transition-related stress were found, although an interaction between transitionrelated stress and avoidant coping was evident, $\beta=.11, t(1,324)=2.03, p<.05$. Probing of this interaction showed a stress-exacerbating effect of avoidant coping on the relation between transition-related stress and young adult substance use $(\beta=-.00, t=.03, p=n s$ at low levels of avoidant coping and $\beta=.19, t=2.49, p=.01$ at high levels of avoidant coping).

In the drug use model, younger emerging adults, $\beta=-.15, t(1,324)=-2.61, p<.001$, and those who reported greater substance use at Wave $3, \beta=.19, t(1,324)=3.32, p<.001$ or greater major life stress at Wave $4, \beta=.19, t(1,324)=3.53, p<.001$, also reported more frequent drug use than their peers. Two interactions were also of note as being significant, involving major life stress and active coping, $\beta=-.12, t(1,324)=-2.02, p<.05$, or marginally significant, involving major life stress and avoidant coping, $\beta=-.10, t(1,324)=$ $-1.71, p<.10$. Probing of these interactions revealed similar effects of active and avoidant coping on the relation between major life events and drug use. A stress-buffering effect showed a significant association between major life events and drug use at low levels of active coping, $\beta=.30, t(1,324)=3.90, p<.001$, and avoidant coping, $\beta=.27, t(1,324)=$ $3.32, p<.001$, but not at high levels of active coping, $\beta=.08, t(1,324)=1.01$, $n s$, or avoidant coping, $\beta=.12, t(1,324)=1.55$, ns.

\section{Discussion}

The current study posited that the development of adequate coping skills in adolescence would better prepare emerging adults to successfully negotiate the stressors and avoid the risky behaviors associated with the transition to adulthood. Using longitudinal data gathered from 11- to 23-year-olds, we examined the development of coping over adolescence and the impact of coping on stress and substance use in young adulthood within a high-risk sample of COAs. Our findings provide moderate support for our hypotheses, and suggest a complex role for coping styles in the relation between stress and substance misuse in young adulthood. Implications of these findings are discussed in turn below.

\section{The development of coping}

We tested the development of coping in two ways. First, we examined intraindividual as well as interindividual change in coping across adolescence. Small to moderate effect sizes suggested significant interindividual continuity in coping over time, such that those higher than their peers in planful or cognitive coping at one point in time were likely to be higher than their peers at another point in time as well. However, our LTMs also showed both significant intraindividual change over time in the normative trends in coping and interindividual differences among adolescents in their coping trajectories. Individual trajectories of both forms of coping showed mean decrements over time, suggesting an average decrease in both planful and cognitive coping over adolescence. Decrements in planful coping over time are consistent with previous support for decreases in approach or behavioral coping over adolescence (Compas et al., 1991). In contrast to our findings, previous studies generally show an increase in cognitive coping over time (Losoya et al., 1998). However, our measure of cognitive coping appears to more closely resemble avoidant coping, for which developmental trends over adolescence have been more inconsistent (Losoya et al., 1998). Based on previous literature, we would have expected to see increases in coping over time if we had more clearly assessed cognitive mechanisms such as cognitive restructuring, reappraisals, positive self-talk, intrapsychic coping, and 
attention diversion that draw on the cognitive advances that typify adolescent development (Ayers et al., 1998; Losoya et al., 1998). Cognitive coping is also thought to develop later than planful approach or more behavioral forms of coping because it is less observable and thus less open to observational learning in childhood. As such, with the development of cognitive coping skills in adolescence, we may see less reliance on the planful and avoidant strategies that is more typical of the coping repertoire of children, resulting in the decreasing trajectories of adolescent planful and avoidant coping found in the current study.

Our second test of the development of coping assessed relations between the adolescent trajectories of coping and active, avoidant, and cognitive coping in young adulthood. Despite a change in measurement and a 5-year lag between our adolescent and adult assessments, adolescent coping continued to modestly predict coping styles in young adulthood. Adolescent planful coping predicted greater active coping in adulthood, whereas adolescent avoidant cognitive coping predicted greater avoidant coping in adulthood. Neither form of adolescent coping predicted adult cognitive coping, again suggesting that our measure of adolescent avoidant cognitive coping is perhaps more heavily avoidant than cognitive in nature.

Fairly consistent relations across the coping styles were also found such that planful coping in adolescence predicted lower avoidant coping in adulthood. In contrast to this inverse prospective prediction, cross-sectional relations among the dimensions of coping in adulthood and adolescence suggested positive associations between adolescent planful and cognitive coping and between cognitive and active coping as well as cognitive and avoidant coping in adulthood. These effects may indicate that within time assessments of coping may better indicate overall levels of coping effort, such that those who are stressed at one point in time are more likely to use all coping strategies. However, over time coping assessments may differentiate the relative styles of coping that an individual favors, such that the relative use of active to avoidant strategies changes over time even though these strategies may all be employed to some extent in the face of a stressor encountered within a given point in time. Although it is tempting to conclude that these dimensions of coping show different cross-sectional and prospective relations, differences in the measurement strategy in adolescence and adulthood must also be considered. These findings more clearly underscore the call from numerous researchers to identify the key dimensions of coping to understand their development and the relation among these dimensions over time (e.g., Compas et al., 2001).

\section{Adolescent coping and young adult adjustment}

We hypothesized that adolescents who enter young adulthood equipped with greater coping skills should also have greater confidence in their ability to successfully respond to stress, and that the resulting skills and confidence should reduce their subjective experience of stress as well as limit their exposure to stressors. These effects of coping on stress should, in turn, reduce risk for heavy alcohol and drug use in young adulthood. This hypothesis was partially supported such that adolescent planful coping, although not cognitive-avoidant coping, predicted substance use in young adulthood. As seen in previous studies, planful coping reduced risk for both heavy alcohol use and drug use in young adulthood (Wills, 1986; Wills et al., 2001; Windle \& Windle, 1996). However, given that neither form of adolescent coping impacted the ability to avoid major life events nor the experience of stress in new adult roles, stress in the transition to adulthood is not likely to explain the relation between planful coping and substance use. Another likely mechanism is impulsivity. Although little research has explored the relation between impulsivity and coping, a consistent and strong relation between impulsivity and substance use has emerged in the literature (Sher, Trull, Bartholow, \& Vieth, 1999). If impulsivity interferes with the cognitive and behavioral tasks of planful coping, decrements in planful coping may signal 
risk for substance use in adulthood associated with impulsivity such as disregard for the potential negative consequences of substance use, seeking out social relationships that create risk for substance use, and impaired skill in avoiding high-risk situations. Further consideration of the relations among coping, impulsivity, and substance use is needed to explore this possible mechanism.

Although not the focus of this study, we found no unique effects of adolescent substance use on later adult coping. Some researchers (Baumrind \& Moselle, 1985) have suggested that adolescent substance use may interfere with the development of mature coping skills by allowing adolescents to avoid rather than learn to effectively adapt to environmental demands. It is possible that the levels of adolescent substance use captured by our measure did not capture the intensity and frequency of use that would be necessary to impair the development of coping in these adolescents. Alternatively, coping skills may be developed sufficiently in adolescence that they are not responsive to the effects of substance use in young adulthood. Perhaps an earlier period of sensitivity within the development of coping would reveal this hypothesized effect.

\section{Stress-buffering and stress-exacerbating effects of coping in young adulthood}

We distinguished between negative, major life events, and transition-related stress in the prediction of young adult substance use. Supporting the uniqueness of these two forms of stress, we found low correlations between these measures. Although the dimensions that most meaningfully differentiate major life events and transition-related stress (i.e., controllability, daily hassles vs. major events, social embeddedness) have yet to be explored, both indicators appear to tap a distinct aspect of stress in this transition. Major life events in adolescents also differentially predicted this form of stress in young adulthood, showing a positive association with major life events in young adulthood but no association with transition-related stress. As such, risk for even uncontrollable, major life events appears to be related to a history of experiencing such events, whereas the adult transition-related stressors do not evolve out of major, negative life events in adolescence. The unique predictors of transition stress may instead come from the potential for all normal adult roles to be stressful, with variability in these roles more a function of such personality constructs as a preformed sense of mastery of internal locus of control upon entering young adulthood. Regardless of differences in their relation to adolescent major life events, young adult major life events and transition-related stress showed independent contributions to the prediction of substance use. These unique contributions were evident in interaction with the moderating effects of both active and avoidant coping.

The current findings are similar to previous literature in showing that active coping buffered the relation between major life events and drug use in adulthood (Wills et al., 2001). Mounting evidence suggests that active forms of coping are generally a successful strategy (Compas et al., 2001). However, two limitations of this finding in the current study should be noted. First, a parallel interaction was not found for the prediction of heavy alcohol use. One possible explanation for this inconsistency is that the stress-coping model underlying this effect may be most relevant for more deviant forms of substance use, with drug use being less normative than heavy alcohol use in young adulthood (Cooper et al., 1995). Second, this buffering effect did not extend to transition-related stress as a risk factor for heavy alcohol or drug use.

Rather, the relation between transition-related stress and alcohol use was exacerbated by greater avoidant coping. Thus, avoidance appears to be a detrimental strategy for coping with the stressors associated with the new roles of adulthood. Many transition-related stressors have some aspect of controllability, suggesting that they may be unlikely to resolve with avoidance. This interpretation is consistent with Lazarus and Folkman (1984), who 
suggest that controllable stress is best addressed with active coping. Unlike coping with a major uncontrollable life event, problems with work, school, relationships, and children may not resolve without more direct, active coping efforts. As such, these stressors may resemble daily hassles, with the potential to become chronic if unchecked by active coping strategies. Moreover, avoidance may perpetuate stress, decrease opportunity for developmental gains in terms of role mastery, and increase risk for seeking out escape activities like substance use that directly provide an avenue for avoidance. Together, these results for transition-related stress suggest that avoidant coping is a poor strategy for addressing this form of stress, but what remains unclear is what forms of coping are most beneficial in terms of mitigating risk for substance involvement. Alternative forms of coping, perhaps that refine the generally more protective domain of active coping, should be examined as moderators of the relation between transition-related stress and substance involvement.

Avoidant coping, however, did not emerge as a uniformly poor coping style in the current study, as a marginally significant interaction showed that the relation between major life events and drug use was buffered by greater avoidant coping. Because the current measure of major life events selected uncontrollable events, these findings again further support Lazarus and Folkman's (1984) proposition that emotional and avoidant coping may be more useful for uncontrollable as opposed to controllable stressors. This finding underscores the importance of considering differences across the domains of stress confronting young adults in determining the impact of coping on adjustment. The distinction between major life events and transition-related stressors suggested here is one more step in this direction.

\section{Parent alcoholism as a risk factor}

Effects of parental alcoholism on coping were only of marginal significance across analyses, resulting in a pattern of weak effects suggesting that COAs may subtlety differ from their peers in the development of coping. COAs reported less planful coping and more cognitiveavoidant coping in adolescence as well as less active coping in young adulthood. With respect to the buffering effects of coping on the relations between stress and substance use, these coping effects generally suggest deficits in COAs'coping compared with their peers. These deficits may in part account for COAs' risk for a variety of negative outcomes, both in adolescence and young adulthood (Chassin et al., 1991; Sher, 1991). Moreover, these coping deficits are likely more pronounced by the greater experience of major life events among COAs across development (as found here and in Chassin et al., 1991; Sher, 1991). COAs did not, however, report greater transition-related stress than their peers. These lack of differences associated with parent alcoholism is surprising, although low power notoriously associated with the detections of three-way interactions may be on explanation for the null finding (Aiken \& West, 1991). Alternatively, differences in role occupancy may create qualitative differences in transition stress not captured by these stress severity ratings. For example, in the current study, COAs were less likely to be students or in the work force than were controls, but they were more likely to be parents and married. As such, COAs may have different experiences in these adult roles than do their peers, and indeed, previous research shows that COAs have more difficulty with leaving the parental home, another important marker in the transition to adulthood (Hussong \& Chassin, 2002). Further examination of transition stress that takes into account differences in role occupancy is clearly needed.

\section{Conclusions}

Overall, the current study supports both continuity and change in coping over development, with the coping skills of adolescence developing into those of adulthood where they impact adjustment. Although these findings are bolstered by the use of a longitudinal, high-risk design and the examination of interindividual and intraindividual change over time, 
limitations of the study should also be considered, such as too few time points to examine nonlinear trajectories of coping over adolescence, reliance on a single reporter for measures of coping, stress, and substance use, and cross-sectional analyses of the stress-buffering and stress-exacerbating models. Nonetheless, these findings suggest that individuals differ both in their levels and patterns of development in coping. These differences provide a possible point for intervening in risk for young adult alcohol and drug use. Moreover, these findings suggest that intervention and prevention efforts should be guided by a focus on the function of coping with respect to various domains of stress that create risk for substance misuse rather than by assuming that certain coping styles are universally more optimal than are others. Such interventions also provide an opportunity for experimentation to better understand the relation between stress in the young adult transition and substance involvement across varying levels of coping styles. Finally, such interventions may elucidate those instances in which acquiring adult roles may actually exacerbate risk for substance use in the face of a normative trend for such roles to dampen this risk.

\section{References}

Aiken, LS.; West, SG. Multiple regression: Testing and interpreting interactions. Newbury Park, CA: Sage; 1991.

Andreasen NC, Endicott J, Spitzer RL, Winokur G. The family history method using diagnostic criteria: Reliability and validity. Archives of General Psychiatry. 1977; 34:1229-1235. [PubMed: 911222]

Arnett JJ. Emerging adulthood: A theory of development from the late teens through the twenties. American Psychologist. 2000; 55:469-480. [PubMed: 10842426]

Arnett JJ. Conceptions of the transition to adulthood: Perspectives from adolescence through midlife. Journal of Adult Development. 2001; 8:133-143.

Ayers, TS.; Sandler, IN.; Twohey, JL. Conceptualization and measurement of coping in children and adolescents. In: Ollendick, TH.; Prinz, RJ., editors. Advances in clinical child psychology. Vol. Vol. 20. New York: Plenum Press; 1998. p. 243-301.

Bachman, JG.; Wadsworth, KN.; O’Malley, PM.; Johnston, LD.; Schulenberg, JE. Smoking, drinking and drug use in young adulthood: The impacts of new freedoms and new responsibilities. Mahwah, NJ: Erlbaum; 1997.

Baer PE, Garmezy LB, McLaughlin RJ, Pokorny AD, Wernick MJ. Stress, coping, family conflict, and adolescent alcohol use. Journal of Behavioral Medicine. 1987; 10:449-466. [PubMed: 3430588]

Baumrind D, Moselle KA. A developmental perspective on adolescent drug abuse. Advances in Alcohol and Substance Abuse. 1985; 4:41-67. [PubMed: 4013874]

Bentler PM. Comparative fit indexes in structural models. Psychological Bulletin. 1990; 107:238-246. [PubMed: 2320703]

Browne, MW.; Cudeck, R. Alternative ways of assessing model fit. In: Bollen, K.; Long, K., editors. Testing structural equation models. Thousand Oaks, CA: Sage Publications; 1993. p. 136-162.

Carbery J, Burhmester D. Friendship and need fulfillment during three phases of young adulthood. Journal of Social and Personal Relationships. 1998; 15:393-409.

Carver CS, Scheier MF, Weintraub JK. Assessing coping strategies: A theoretically based approach. Journal of Personality and Social Psychology. 1989; 56:267-283. [PubMed: 2926629]

Chassin L, Barrera M, Bech K, Kossak-Fuller J. Recruiting a community sample of adolescent children of alcoholics. Journal of Studies on Alcohol. 1992; 53:316-319. [PubMed: 1619925]

Chassin L, Curran PJ, Hussong AM, Colder CR. The relation of parent alcoholism to adolescent substance use: A longitudinal follow-up study. Journal of Abnormal Psychology. 1996; 105:7080. [PubMed: 8666713]

Chassin L, Pitts SC, DeLucia C, Todd M. A longitudinal study of children of alcoholics: Predicting young adult substance use disorders, anxiety, and depression. Journal of Abnormal Psychology. 1999; 108:106-119. [PubMed: 10066997] 
Chassin LA, Rogosch R, Barrera M. Substance use and symptomatology among adolescent children of alcoholics. Journal of Abnormal Psychology. 1991; 100:449-463. [PubMed: 1757658]

Cicchetti, D.; Rogosch, FA.; Toth, SL. Ontogenesis, depressotypic organization, and the depressive spectrum. In: Luthar, SS.; Burack, JA.; Cicchetti, D.; Weisz, JR., editors. Developmental psychopathology: Perspectives on adjustment, risk and disorder. New York: Cambridge University Press; 1997. p. 273-313.

Compas BE, Banez GE, Malcarne V, Worsham N. Perceived control and coping with stress: A developmental perspective. Journal of Social Issues. 1991; 47:23-34.

Compas BE, Connor-Smith JK, Saltzman H, Thomsen AH, Wadsworth ME. Coping with stress during childhood and adolescence: Problems, progress, and potential in theory and research. Psychological Bulletin. 2001; 127:87-127. [PubMed: 11271757]

Compas BE, Howell DC, Phares V, Williams RA, Giunta CT. Risk factors for emotional/behavioral problems in young adolescents: A prospective analysis of adolescent and parental stress and symptoms. Journal of Consulting and Clinical Psychology. 1989; 57:732-740. [PubMed: 2600244]

Cooper ML, Frone MR, Russell M, Mudar P. Drinking to regulate positive and negative emotions: A motivational model of alcohol use. Journal of Personality and Social Psychology. 1995; 69:9901005. [PubMed: 7473043]

Cooper ML, Russell M, George WH. Coping, expectancies, and alcohol abuse: A test of social learning formulations. Journal of Abnormal Psychology. 1988; 97:218-230. [PubMed: 3385075]

Cooper ML, Russell M, Skinner JB, Frone MR, Mudar P. Stress and alcohol use: Moderating effects of gender, coping and alcohol expectancies. Journal of Abnormal Psychology. 1992; 101:139-152. [PubMed: 1537960]

DeLongis A, Coyne JC, Dakof G, Folkman S, Lazarus R. Relationship of daily hassles, uplifts, and major life events to health status. Health Psychology. 1982; 1:119-136.

Easley MJ, Epstein N. Coping with stress in a family with an alcoholic parent. Family Relations. 1991; 40:218-224.

Eccles, J.; Templeton, J.; Barber, B.; Stone, M. Adolescence and emerging adulthood: The critical passage ways to adulthood. In: Bornstein, MH.; Davidson, L.; Keye, CLM.; Moore, KA., editors. Wellbeing: Positive development across the life course. Mahwah, NJ: Erlbaum; 2003. p. 383-406.

Fromme K, Rivet K. Young adults' coping styles as a predictor of their alcohol use and response to daily events. Journal of Youth and Adolescence. 1994; 23:85-97.

Gotham HJ, Sher KJ, Wood PK. Alcohol involvement and developmental task completion during young adulthood. Journal of Studies on Alcohol. 2003; 64:32-42. [PubMed: 12608481]

Hussong AM, Chassin L. Parent alcoholism and the leaving home transition. Developmental Psychopathology. 2002; 14:139-157.

Jarmas AL, Kazak AE. Young adult children of alcoholic fathers: Depressive experiences, coping styles, and family systems. Journal of Consulting and Clinical Psychology. 1992; 60:244-251. [PubMed: 1592954]

Johnson V, Pandina R. A longitudinal examination of the relationships among stress, coping strategies, and problems associated with alcohol use. Alcoholism: Clinical and Experimental Research. 1993; 17:696-702.

Kelly VA, Myers JE. Parental alcoholism and coping: A comparison of female children of alcoholics with female children of nonalcoholics. Journal of Counseling and Development. 1997; 74:501504.

Kosten TA, Anton SF, Rounsaville BJ. Ascertaining psychiatric diagnoses with the family history method in a substance abuse population. Journal of Psychiatric Research. 1992; 26:135-147. [PubMed: 1613680]

Laurent J, Catanzaro SJ, Callan MB. Stress, alcohol-related expectancies and coping preferences: A replication with adolescents of the Cooper et al. (1992) Model. Journal of Studies on Alcohol. 1997; 58:644-651. [PubMed: 9391925]

Lazarus, RS.; Folkman, S. Stress, appraisal and coping. New York: Springer; 1984.

Lerner, RM.; Lerner, JV.; von Eye, A.; Ostrum, CW.; Nitz, K.; Talwar-Soni, R.; Tubman, J. Continuity and discontinuity across the transition of early adolescence: A developmental 
contextual perspective. In: Graber, JA.; Brooks-Gunn, J.; Petersen, AC., editors. Transitions through adolescence: Interpersonal domains and contexts. Mahwah, NJ: Erlbaum; 1996. p. 3-22.

Loehlin, JC. Latent variable models: An introduction to factor, path, and structural analysis. Hillsdale, NJ: Erlbaum; 1992.

Losoya S, Eisenberg N, Fabes RA. Developmental issues in the study of coping. International Journal of Behavioral Development. 1998; 22:287-313.

Maisto, SA.; Carey, KB.; Bradizza, CM. Social learning theory. In: Leonard, KE.; Blane, HT., editors. Psychological theories of drinking and alcoholism. 2nd ed. New York: Guilford Press; 1999. p. 106-163.

Menees MM. The role of coping, social support, and family communication in explaining the selfesteem of adult children of alcoholics. Communication Reports. 1997; 10:9-19.

Newcomb MD. Pseudomaturity among adolescents: Construct validation, sex differences, and associations in adulthood. Journal of Drug Issues. 1996; 26:477-504.

Newcomb MD, Bentler PM. The impact of high school substance use on choice of young adult living environment and career directions. Journal of Drug Education. 1985; 15:253-261. [PubMed: 4078654]

Newcomb MD, Bentler PM. Drug use, educational aspirations, and workforce involvement: The transition from adolescence to young adulthood. American Journal of Community Psychology. 1986; 14:303-321. [PubMed: 3739981]

Newcomb, MD.; Bentler, PM. Consequences of adolescent drug use: Impact on the lives of young adults. Beverly Hills, CA: Sage; 1988.

Robins LN, Helzer JE, Croughan J, Ratcliff KS. National Institute of Mental Health Diagnostic Interview Schedule: Its history, characteristics, and validity. Archives of General Psychiatry. 1981; 38:381-389. [PubMed: 6260053]

Roosa MW, Sandler IN, Gehring M, Beals J, Cappo L. The children of alcoholics life-events schedule: A stress scale for children of alcohol-abusing parents. Journal of Studies on Alcohol. 1988; 49:422-429. [PubMed: 3216645]

Sameroff, AJ. Dialectical processes in developmental psychopathology. In: Sameroff, AJ.; Lewis, M.; Miller, SM., editors. Handbook of developmental psychopathology. 2nd ed. New York: Plenum Press; 2000. p. 23-40.

Sandler, IN.; Ramirez, R.; Reynolds, K. Life stress for children of divorce, bereaved and asthmatic children; Paper presented at the Annual Meeting of the American Psychological Association; Washington, DC. 1986.

Sandler IN, Tein JY, West SG. Coping, stress and the psychological symptoms of children of divorce: A cross-sectional and longitudinal study. Child Development. 1994; 65:1744-1763. [PubMed: 7859552]

Sandler, IN.; Wolchik, SA.; MacKinnon, D.; Ayers, TS.; Roosa, MW. Developing linkage between theory and intervention in stress and coping processes. In: Wolchik, SA.; Sandler, IN., editors. Handbook of children's coping: Linking theory and intervention. New York: Plenum Press; 1997. p. 3-40.

Schwartz JE, Pieper CF, Karasek RA. A procedure for linking psychosocial job characteristics Stress and coping data to health surveys. American Journal of Public Health. 1988; 78:904-909. [PubMed: 3389426]

Sher, KJ. Children of alcoholics: A critical appraisal of theory and research. Chicago: University of Chicago Press; 1991.

Sher, KJ.; Trull, TJ.; Bartholow, BD.; Vieth, A. Personality and alcoholism: Issues, methods, and etiological processes. In: Leonard, K.; Blane, HT., editors. Psychological theories of drinking and alcoholism. New York: Guilford Press; 1999. p. 54-105.

Sher KJ, Walitzer KS, Wood PK, Brent EE. Characteristics of children of alcoholics: Putative risk factors, substance use and abuse, and psychopathology. Journal of Abnormal Psychology. 1991; 100:427-448. [PubMed: 1757657]

Steiger, JH.; Lind, JC. Statistically based tests for the number of common factors; Paper presented at the annual meeting of the Psychometric Society; Iowa City, IA. 1980 May. 
Stein JA, Smith GM, Guy SM, Bentler PM. Consequences of adolescent drug use on young adult job behavior and job satisfaction. Journal of Applied Psychology. 1993; 78:463-474. [PubMed: 8331025]

Todd M, Chassin L, Presson CC, Sherman SJ. Role stress, role socialization, and cigarette smoking: Examining multiple roles and moderating variables. Psychology of Addictive Behaviors. 1996; 10:211-221.

Tucker LR, Lewis C. A reliability coefficient for maximum likelihood factor analysis. Psychometrika. 1973; 38:1-10.

Wagner BM, Compas BE, Howell DC. Daily and major life events: A test of an integrative model of psychosocial stress. American Journal of Community Psychology. 1988; 16:189-205. [PubMed: 3407632]

Wills, TA. Supportive functions of interpersonal relationships. In: Cohen, S.; Syme, SL., editors. Social support and health. Orlando, FL: Academic Press; 1985. p. 61-78.

Wills TA. Stress and coping in early adolescence: Relationships to substance use in urban school samples. Health Psychology. 1986; 5:503-529. [PubMed: 3492372]

Wills, TA. Coping processes and self-efficacy: Prospective analyses in cohorts of urban adolescents. Bronx, NY: Ferkauf Graduate School of Psychology and Albert Einstein Medical School; 1989. Unpublished manuscript

Wills TA, Sandy JM, Yaeger AM, Cleary SD, Shinar O. Coping dimensions, life stress, and adolescent substance use: A latent growth analysis. Journal of Abnormal Psychology. 2001; 110:309-323. [PubMed: 11358025]

Windle M, Windle RC. Coping strategies, drinking motives, and stressful life events among middle adolescents: Associations with emotional and behavioral problems and with academic functioning. Journal of Abnormal Psychology. 1996; 105:551-560. [PubMed: 8952188]

Zautra AJ, Sheets VL, Sandler IN. An examination of the construct validity of coping dispositions for a sample of recently divorced mothers. Psychological Assessment. 1996; 8:256-264.

Zimmerman M, Coryell W, Pfohl B, Stangl D. The reliability of the family history method for psychiatric diagnoses. Archives of General Psychiatry. 1988; 45:320-322. [PubMed: 3281625] 


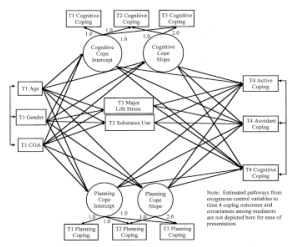

Figure 1.

Estimated latent trajectory model predicting young adult coping. 


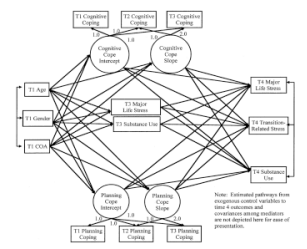

Figure 2.

Estimated latent trajectory model predicting young adult stress and substance use. 
Table 4

Regression models testing stress-buffering and stress-exacerbating hypotheses

\begin{tabular}{lrrrrr}
\hline & \multicolumn{2}{c}{$\begin{array}{c}\text { Time 4 Heavy } \\
\text { Alcohol Use Model }\end{array}$} & \multicolumn{2}{c}{$\begin{array}{c}\text { Time 4 Drug } \\
\text { Use Model }\end{array}$} \\
\cline { 2 - 3 } Predictors & $\boldsymbol{\beta}$ & $\boldsymbol{t}$ Value & & $\boldsymbol{\beta}$ & $\boldsymbol{t}$ Value \\
\hline Age & .01 & 0.24 & -.15 & $-2.61^{* * *}$ \\
Gender & .21 & $4.21^{* * *}$ & .07 & 1.30 \\
COA & .13 & $2.46^{* *}$ & .03 & 0.50 \\
Time 3 substance use & .28 & $5.23^{* * *}$ & .19 & $3.32^{* * *}$ \\
Time 4 & & & & \\
MLS & .13 & $2.47^{* *}$ & .19 & $3.53^{* * *}$ \\
TRS & .08 & 1.62 & .04 & 0.66 \\
ACC & -.07 & -1.29 & -.09 & -1.51 \\
CC & .06 & 1.14 & .04 & 0.65 \\
AVC & -.01 & -0.08 & -.03 & -0.51 \\
MLS $\times$ ACC & -.05 & -0.95 & -.12 & $-2.02^{*}$ \\
MLS $\times$ CC & .05 & 0.95 & .03 & 0.48 \\
MLS $\times$ AVC & -.05 & -0.85 & -.10 & $-1.71^{\dagger}$ \\
TRS $\times$ ACC & .07 & 1.18 & .01 & 0.15 \\
TRS $\times$ CC & -.04 & -0.67 & -.04 & -0.69 \\
TRS $\times$ AVC & .11 & $2.03^{*}$ & .04 & 0.74 \\
\hline
\end{tabular}

Note: Regression parameter estimates are standardized betas. MLS, major life stress; TRS, transition-related stress; ACC, active coping; CC, cognitive coping; AVC, avoidant coping.

The $t$ values are significant at

${ }^{\dagger} p<.10$,

* $p<.05$,

** $p<.01$, and

*** $p<.001$ with $d f=1,324$. 